\title{
Identifying the Gene Signatures from Gene-Pathway Bipartite Network Guarantees the Robust Model Performance on Predicting the Cancer Prognosis
}

\author{
Li He, ${ }^{1}$ Yuelong Wang, ${ }^{2}$ Yongning Yang, ${ }^{2}$ Liqiu Huang, ${ }^{2}$ and Zhining Wen ${ }^{2}$ \\ ${ }^{1}$ Biogas Institute of Ministry of Agriculture, Chengdu 610041, China \\ ${ }^{2}$ College of Chemistry, Sichuan University, Chengdu 610064, China \\ Correspondence should be addressed to Zhining Wen; w_zhining@163.com
}

Received 16 April 2014; Revised 21 June 2014; Accepted 24 June 2014; Published 14 July 2014

Academic Editor: Mingyue Zheng

Copyright (C) $2014 \mathrm{Li} \mathrm{He}$ et al. This is an open access article distributed under the Creative Commons Attribution License, which permits unrestricted use, distribution, and reproduction in any medium, provided the original work is properly cited.

For the purpose of improving the prediction of cancer prognosis in the clinical researches, various algorithms have been developed to construct the predictive models with the gene signatures detected by DNA microarrays. Due to the heterogeneity of the clinical samples, the list of differentially expressed genes (DEGs) generated by the statistical methods or the machine learning algorithms often involves a number of false positive genes, which are not associated with the phenotypic differences between the compared clinical conditions, and subsequently impacts the reliability of the predictive models. In this study, we proposed a strategy, which combined the statistical algorithm with the gene-pathway bipartite networks, to generate the reliable lists of cancer-related DEGs and constructed the models by using support vector machine for predicting the prognosis of three types of cancers, namely, breast cancer, acute myeloma leukemia, and glioblastoma. Our results demonstrated that, combined with the gene-pathway bipartite networks, our proposed strategy can efficiently generate the reliable cancer-related DEG lists for constructing the predictive models. In addition, the model performance in the swap analysis was similar to that in the original analysis, indicating the robustness of the models in predicting the cancer outcomes.

\section{Introduction}

In the past decade, DNA microarray technology has been widely used in clinical researches to predict the cancer outcomes because of its capability of monitoring tens of thousands of genes simultaneously [1-9]. Accurately identifying the genes, for which the changes of their expression levels are significantly correlated with the phenotypic differences between the clinical conditions, plays an important role in the procedure of clinical model construction. The statistical methods and the machine learning algorithms that are routinely used for gene selection mainly identify the differentially expressed genes (DEGs) according to the changes of the gene expression levels between the compared biological samples. However, because of the heterogeneity of the clinical samples, the changes of the gene expression levels may be not only caused by the changes in the status of the cancer cells but also by those of the cells unrelated to the cancers. In addition, the intensities detected by the microarrays for a gene will vary to some extent among the technical replicates due to the complex procedures of the microarray experiment, such as labeling, hybridization, and scanning. Consequently, the DEG list generated by the statistical methods or the machine learning algorithms often involves a number of false positive genes, which are not associated with the phenotypic differences between the compared clinical samples, and subsequently impacts the reliability of the predictive models.

The network-based methodologies can efficiently integrate the biological information with the computational techniques and link the disease-related genes to relevant proteins and disease types. In recent years, the network-based methodologies have been successfully introduced into the systems biology researches for drug discovering [2], identifying disease-related genes [10-15], and revealing the molecular mechanisms of tumorigenesis [16-19]. In clinical researches, the prediction models constructed with the cancer-related 
gene markers, which were selected only by the statistical methods or the machine learning algorithms, cannot ensure the accuracy and the reproducibility in predicting the clinical outcomes of cancer patients. Therefore, for the sake of improving the model performance and interpreting the biological relevance of the gene markers and the specific cancer, a number of network-based algorithms were developed to prioritize the prognostic genes [20-38].

In our study, a new strategy, which combined the statistical algorithm with a gene-pathway bipartite network, was proposed to prioritize the reliable gene signatures and the supported vector machine (SVM) was used to construct the models for predicting the clinical outcomes of cancer patients. The DEG list was firstly generated by the statistical methods, for example, Student's $t$-test. Then, the bipartite network that connected the genes and the cancer-related pathways was constructed to score each of the DEGs according to its connectivity in the network. Finally, the DEGs were ranked by the scores in descending order and those, for which the scores were greater than a given cutoff, were selected as features for predicting the cancer prognosis.

To evaluate the performance of the predictive models with the gene signatures generated by our strategy, three data sets including the gene expression data of the clinical samples collected from the patients of breast cancer, acute myeloma leukemia, and glioblastoma were downloaded from the gene expression omnibus (GEO) database. Gene signatures separately identified from these data sets by our strategy were used as features to predict the reoperative treatment response of breast cancer, the overall survival milestone outcome of acute myeloma leukemia, and the molecular subclasses of highgrade glioblastoma. The results of predicting the reoperative treatment response of breast cancer and the overall survival milestone outcome of acute myeloma leukemia showed that our models performed better than those reported by the data contributors. In addition, the accuracy of predicting the molecular subclasses of high-grade glioblastoma was as high as $87.5 \%$. In the swap analysis, we repeated the model construction and validation process by training the models with the original independent test set and validating them using the original training set with the same gene signatures prioritized in the original analysis. The prediction results were similar to those achieved in original analysis, indicating the robust model performance on predicting the cancer prognosis when using the gene signatures identified by our proposed strategy.

\section{Materials and Methods}

2.1. Data Sets. All microarray gene expression data (series MATRIX files) generated from the clinical samples of breast cancer, acute myeloma leukemia, and glioblastoma and the corresponding clinical information were downloaded from the National Center for Biotechnology Information's Gene Expression Omnibus (GEO) database (series accession numbers: GSE16716, GSE12417, and GSE13041).

In the human breast cancer data set $[4,9]$, the gene expression data of 230 clinical samples were generated by using Affymetrix Human Genome U133A (HG-U133A) microarrays, which included 22,283 probesets. In light of the data analysis protocol in MicroArray Quality Control (MAQC)-II Project [9], the gene expression data generated from 130 out of 230 clinical samples of breast cancer patients were used as training set and the rest of the 100 cases were used as independent test set. The response to preoperative chemotherapy, which was divided into two subcategories of no residual invasive cancer in the breast or lymph nodes ( $\mathrm{pCR}$ ) and residual invasive cancer (RD), was used as the clinical endpoint for prediction [4].

The acute myeloma leukemia data set included the gene expression profiling of the clinical samples of 242 patients with cytogenetically normal acute myeloid leukemia $(\mathrm{CN}-$ AML) [39]. In the training set, the gene expression data of 163 clinical samples were generated by using Affymetrix Human Genome U133A\&B (HG-U133A\&B) microarrays, which included a total of 44,760 probesets. The gene expression data of 79 clinical samples in the independent test set were generated by using HG-U133Plus2 microarrays. During the calculation procedure, we only used 44,693 common probesets between HG-U133A\&B chips and HGU133Plus2 chips for the DEG selection and predictive model construction. The clinical endpoint of overall survival times was dichotomized with a "milestone" cutoff because the continuous endpoint values cannot be predicted by the binary classification models. By considering the balance between the number of positive samples and that of negative samples, the patients with the survival time less than one year were categorized into the "high-risk" group and the rest with the survival time equal to or longer than one year were assigned to the "low-risk" group. In addition, a patient was excluded from the data set if the survival time was less than one-year milestone cutoff and censored when he/she was still alive. Eventually, there were 152 patients in the training set and 77 patients in the independent test set.

The gene expression data in the glioblastoma data set [7] were generated by using HG-U133A microarrays. In glioblastoma research, a subcategory of glioblastoma termed ProNeural (PN) was highly related to better survival prognosis when compared to other subcategories [6]. In our study, we collected 50 patients belonging to the $\mathrm{PN}$ subcategory with a mean survival of 924 days and 50 patients belonging to nonPN subcategory with a mean survival of 150 days. Among these patients, 60 of them, which included 30 patients in PN subcategory and 30 in non-PN subcategory, were randomly assigned to the training set and the rest were used as the independent test set. A predictive model was constructed to discriminate the $\mathrm{PN}$ and non-PN categories based on the microarray gene expression data.

2.2. Probesets Mapping. For Affymetrix microarray platforms, a gene may be detected by multiple probesets. Before identifying DEGs, we mapped the multiple probesets to a unique HUGO gene symbol by using the probeset with the highest fold change value between two groups of samples. Accordingly, 22,283 probesets involved in the data sets of breast cancer and glioblastoma were mapped to 11,285 unique 
genes and 44,693 common probesets involved in the acute myeloma leukemia data set were mapped to 14,892 unique genes, respectively.

2.3. Identification of Differentially Expressed Genes. Student's $t$-test, which can assess how significant a gene is differentially expressed in two compared phenotypes, was used in our study for the DEG selection. The $P$ value for each of the genes was calculated by $t$-test and directly used for gene filtering without multiple-testing correction. Only the genes with $P<$ 0.05 were kept. To ensure the reproducibility of the DEG lists generated by the $t$-test, a fold change ranking is usually applied to refining the genes with $P<0.05$. These genes were ranked by their fold changes (the expression intensity of a gene in sample A/its expression intensity in sample B). Only the genes with fold change $>1.5(\mathrm{FC}>1.5)$ or fold change $<0.667$ (FC < 0.667) were kept for the subsequent analysis. Note that, in some microarray studies of clinical samples, only a few genes can meet the fold change cutoff because of the minor phenotypic differences between the two groups of clinical samples.

2.4. Construction of Gene-Pathway Bipartite Network. For the purpose of screening out the genes unassociated with the phenotypic differences, we constructed a gene-pathway bipartite network, which can be used to score the genes according to their connections [40] to the cancer-related signaling pathways. All the cancer-related pathways were collected from Kyoto Encyclopedia of Genes and Genomes (KEGG) pathway database and listed in Table 1. The first six pathways reflected the overview of cancers and the rest were correlated with the specific types of cancers.

The bipartite network was a particular class of complex networks, in which the nodes were divided into two groups and the connections only existed between two nodes in different groups [41]. So, the nodes in the gene-pathway bipartite network were genes or pathways and were divided into two groups of gene set and pathway set, respectively. The connections between genes and pathways indicated (1) which genes were involved in a specific pathway and (2) which pathways included a specific gene. We scored each of the genes with a weighting method proposed by Zhou et al. [42]. Let us consider a gene-pathway bipartite network $N(\mathbf{G}, \mathbf{P}, \mathbf{E})$, where $G$ and $P$ represent the gene set and pathway set, respectively. $\mathbf{E}$ is the set of connections between genes and pathways. The genes and pathways in $\mathbf{G}$ and $\mathbf{P}$ were denoted by $g_{1}, g_{2}, \ldots, g_{n}$ and $p_{1}, p_{2}, \ldots, p_{m}$, respectively. The initial score $s_{0}$ assigned to each of the genes in $\mathbf{G}$ was set to 1 . In the first step, we calculated the weights $W(W=$ $\left.\left\{w_{1}, w_{2}, \ldots, w_{m}\right\}\right)$ for the pathways via

$$
w_{l}=\sum_{j=1}^{n} \frac{a_{j l} s_{0}}{k\left(g_{j}\right)}
$$

$(l=1,2, \ldots$, the number of pathways in $P)$, where $k\left(g_{j}\right)$ was the degree of the $j$ th gene and $a_{j l}$ was the $n \times m$ adjacent matrix:

$$
a_{j l}= \begin{cases}1, & g_{j} p_{l} \in E \\ 0, & \text { otherwise }\end{cases}
$$

In the second step, we calculated the final scores $S(S=$ $\left.\left\{s_{1}, s_{2}, \ldots, s_{n}\right\}\right)$ for all the genes via

$$
s_{i}=\sum_{l=1}^{m} \frac{a_{i l} w_{l}}{k\left(p_{l}\right)} \quad(i=1,2, \ldots, \text { the number of genes in } G) \text {, }
$$

where $k\left(p_{l}\right)$ and $w_{l}$ were the degree and the weight of the $l$ th pathway, respectively, and $a_{i l}$ was the $n \times m$ adjacent matrix:

$$
a_{i l}= \begin{cases}1, & g_{i} p_{l} \in E \\ 0, & \text { otherwise }\end{cases}
$$

By combining (1) and (3), we can directly calculate the scores for the genes via

$$
\begin{aligned}
s_{i} & =\sum_{l=1}^{m} \frac{a_{i l}}{k\left(p_{l}\right)} \sum_{j=1}^{n} \frac{a_{j l} s_{0}}{k\left(g_{j}\right)} \\
& =\sum_{j=1}^{n} c_{i j} s_{0} \quad(i=1,2, \ldots, \text { the number of genes in } G),
\end{aligned}
$$

where

$$
c_{i j}=\frac{1}{k\left(g_{j}\right)} \sum_{l=1}^{m} \frac{a_{i l} a_{j l}}{k\left(p_{l}\right)}
$$

The matrix $\mathbf{C}=\left\{c_{i j}\right\}_{n \times n}$ represented the weighted $\mathbf{G}$ projection. In our study, the DEGs were firstly selected by the statistical methods and subsequently ranked by the scores $\mathbf{S}\left(\mathbf{S}=\left\{s_{1}, s_{2}, \ldots, s_{n}\right\}\right)$. Only the DEGs with $s \geq 1$ were kept as features for the construction of the predictive models.

2.5. Model Construction for Clinical Endpoints Prediction. The binary classification models for predicting the clinical endpoints were constructed by using support vector machine (SVM), which is a popular learning machine based on statistical learning theory $[43,44]$. In our study, radial basic function was used as the kernel function in SVM. The regularization parameter $c$ and the kernel width parameter $\sigma$ were optimized by a grid search approach. For each of the clinical endpoints, the SVM model was built by using the training set and leave-one-out cross-validation and validated by the independent test set. Four performance metrics, namely, specificity, sensitivity, accuracy, and Matthew's correlation 
TABLE 1: The 20 cancer-related signaling pathways collected from KEGG database for the construction of gene-pathway bipartite network.

\begin{tabular}{lcc}
\hline Pathway entry & KEGG pathway name & Number of genes \\
\hline hsa05200 & Pathways in cancer & 327 \\
hsa05202 & Transcriptional misregulation in cancer & 179 \\
hsa05203 & Viral carcinogenesis & 206 \\
hsa05204 & Chemical carcinogenesis & 80 \\
hsa05205 & Proteoglycans in cancer & 225 \\
hsa05206 & MicroRNAs in cancer & 296 \\
hsa05210 & Colorectal cancer & 62 \\
hsa05211 & Renal cell carcinoma \\
hsa05212 & Pancreatic cancer & 66 \\
hsa05213 & Endometrial cancer & 66 \\
hsa05214 & Glioma & 52 \\
hsa05215 & Prostate cancer & 65 \\
hsa05216 & Thyroid cancer & 89 \\
hsa05217 & Basal cell carcinoma & 29 \\
hsa05218 & Melanoma & 55 \\
hsa05219 & Bladder cancer & 71 \\
hsa05220 & Chronic myeloid leukemia \\
hsa05221 & Acute myeloid leukemia \\
hsa05222 & Small cell lung cancer \\
hsa05223 & Non-small-cell lung cancer & 38 \\
\hline
\end{tabular}

coefficient (MCC), were considered for model evaluation and defined as follows:

$$
\begin{gathered}
\text { Specificity }=\frac{\mathrm{TN}}{\mathrm{FP}+\mathrm{TN}}, \\
\text { Sensitivity }=\frac{\mathrm{TP}}{\mathrm{TP}+\mathrm{FN}}, \\
\text { Accuracy }=\frac{\mathrm{TP}+\mathrm{TN}}{\mathrm{TP}+\mathrm{FP}+\mathrm{TN}+\mathrm{FN}}, \\
\mathrm{MCC}=(\mathrm{TP} \times \mathrm{TN}-\mathrm{FP} \times \mathrm{FN}) \\
\times((\mathrm{TP}+\mathrm{FP}) \times(\mathrm{TP}+\mathrm{FN}) \\
\quad \times(\mathrm{TN}+\mathrm{FP}) \times(\mathrm{TN}+\mathrm{FN}))^{-1 / 2},
\end{gathered}
$$

where TP, FP, TN, and FN represent true positive, false positive, true negative, and false negative, respectively. In addition, the areas under the ROC curves (AUCs) were also provided for evaluating the performance of the models on the prediction of the survival milestone outcomes of AML patients and the molecular subclasses of high-grade glioblastoma. The software libsvm 3.17 [45] used in our study for SVM modeling can be freely downloaded from the website http://www.csie.ntu.edu.tw/ cjlin/libsvm/.

\section{Results}

3.1. Model Performance on Predicting the Reoperative Treatment Response of Breast Cancer. According to the data analysis protocol in MAQC-II project, 130 clinical samples of breast cancer patients were assigned to training set and the rest of the 100 clinical samples were used as independent test set. By comparing the gene expression profiles of the samples in pCR subcategory with those in $\mathrm{RD}$ subcategory in training set, 1010 genes with $P$ value $<0.05$ and $\left|\log _{2} \mathrm{FC}\right|>0.585$ were selected as DEGs and used to construct the gene-pathway bipartite network. Based on the connections between the DEGs and the cancer-related KEGG pathways, 1010 DEGs were scored by a weighted method and then 29 DEGs with the scores $\geq 1$ were kept as features for model construction. The gene-pathway bipartite network, which connected the 29 DEGs with the 20 cancer-related KEGG pathways, was shown in Figure 1. It can be seen from Figure 1 that the gene CCND1, which was ranked 1st in the 1010 DEGs, had the most connections to the cancer-related pathways, indicating it was an important feature for the prediction of the clinical endpoint of breast cancer.

A SVM model was constructed by using the training set and leave-one-out cross-validation. The best parameters of $c$ and $\sigma$ were 2 and 0.03125 , respectively. The prediction results of training set and independent test set were listed in Table 2. In swap analysis, we repeated the model construction and validation process by training the models with the original independent test set and validating them using the original training set with the same 29 DEGs identified in the original analysis. Meanwhile, the prediction results achieved by MAQC-II candidate models were also listed in Table 2. Compared with the MAQC-II candidate models, our model was more robust and superior in predicting the breast cancer outcomes.

3.2. Model Performance on Predicting the Overall Survival Milestone Outcome of Acute Myeloma Leukemia. By comparing the gene expression profiles of the clinical samples 


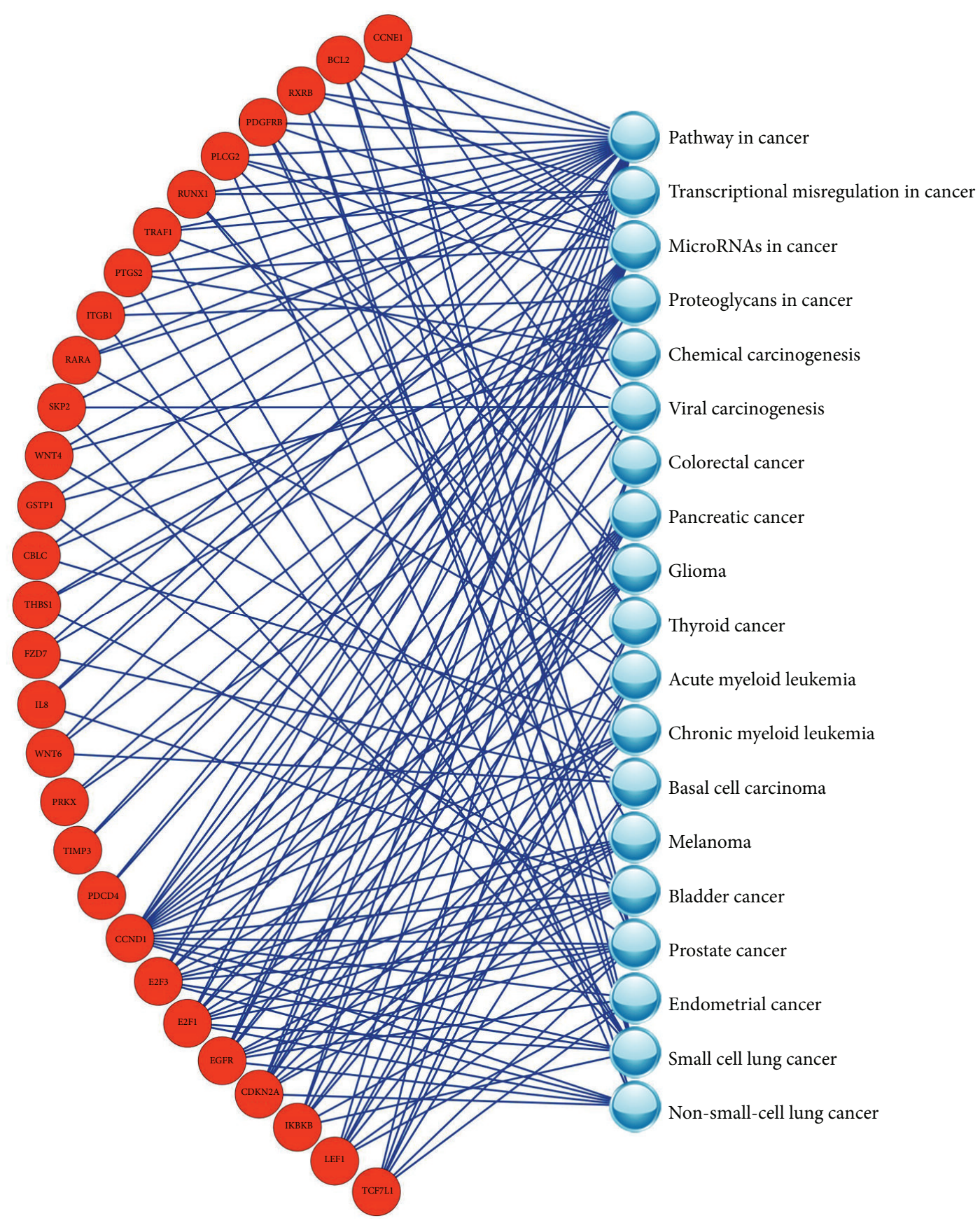

FIGURE 1: The gene-pathway bipartite network constructed with 29 gene signatures that were used for predicting the reoperative treatment response of breast cancer.

TABLE 2: The results of predicting the reoperative treatment response of breast cancer in original and swap analyses.

\begin{tabular}{lccccccrrr}
\hline & & \multicolumn{4}{c}{ Our model } & \multicolumn{4}{c}{ MAQC-II candidate model } \\
& & SP & SE & ACC & MCC & SP & SE & ACC & MCC \\
\hline \multirow{2}{*}{ Original analysis } & Training & 0.928 & 0.455 & 0.808 & 0.444 & 0.847 & 0.569 & 0.775 & 0.433 \\
& Validation & 0.882 & 0.467 & 0.820 & 0.332 & 0.729 & 0.667 & 0.720 & 0.301 \\
\hline \multirow{2}{*}{ Swap analysis } & Training & 0.988 & 0.200 & 0.870 & 0.343 & 0.899 & 0.522 & 0.837 & 0.454 \\
& Validation & 1.000 & 0.152 & 0.785 & 0.343 & 0.959 & 0.212 & 0.769 & 0.267 \\
\hline
\end{tabular}

In the prediction, $\mathrm{pCR}$ was defined as positive sample.

SP, SE, ACC, and MCC represented specificity, sensitivity, accuracy, and Matthew's correlation coefficient, respectively. 


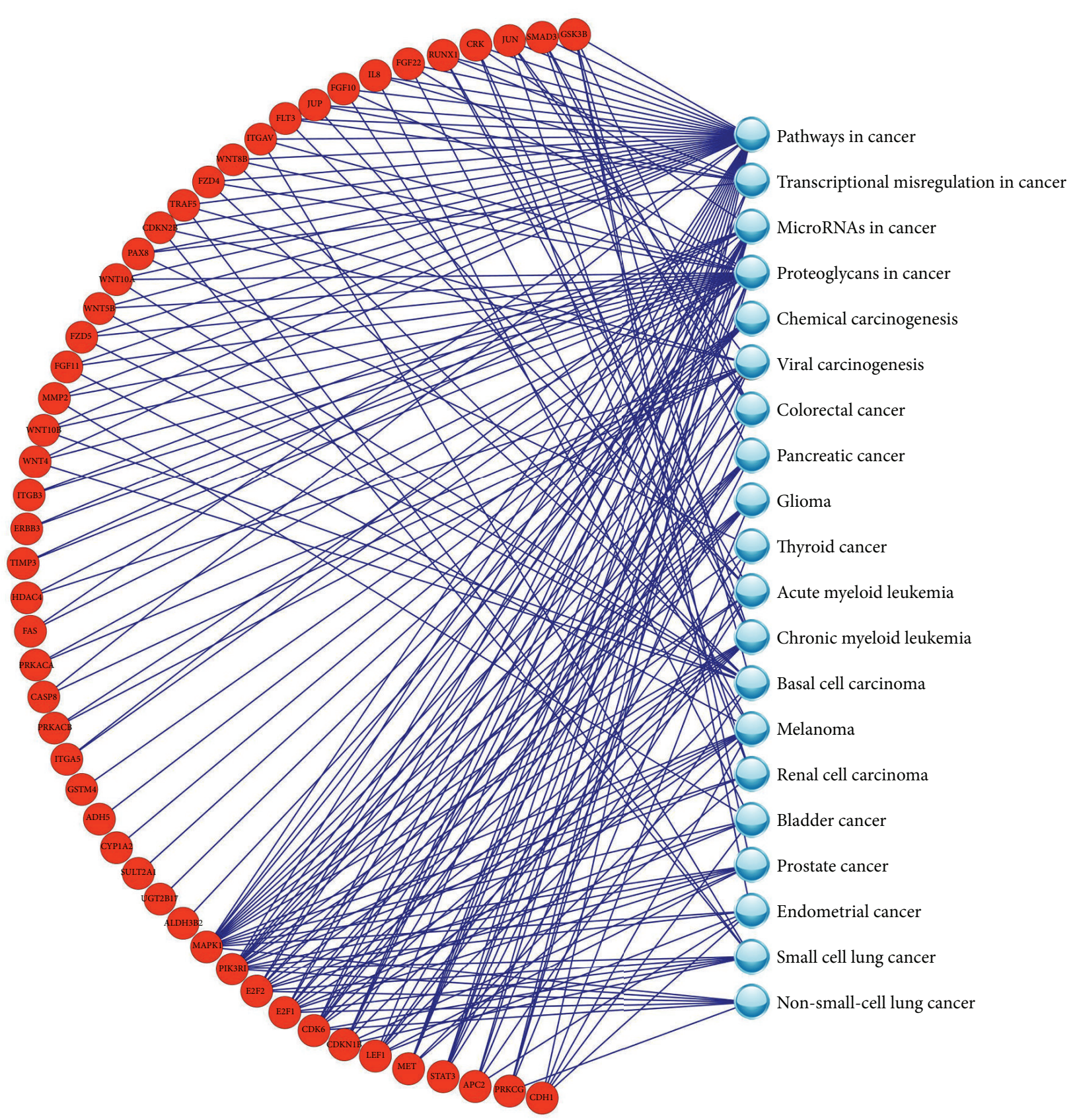

FIGURE 2: The gene-pathway bipartite network constructed with 50 gene signatures that were used for predicting the overall survival milestone outcome of acute myeloma leukemia.

between the high-risk patients and the low-risk patients in the training set, 3234 genes with $P$ value $<0.05$ were selected as DEGs. These DEGs were used to construct the gene-pathway bipartite network and ranked by their scores calculated by the weighted method. At last, 50 DEGs with the scores $\geq 1$ were used as features for the subsequent model construction. The gene-pathway bipartite network of 50 DEGs connected to the 20 cancer-related KEGG pathways was shown in Figure 2. The gene MAPK1 was ranked 1st in the DEG list and had the most connections to the cancer-related pathways.
In both original and swap analyses, the best parameters of $c$ and $\sigma$ optimized for SVM models were 512 and 0.00195 , respectively. The prediction results achieved by our models were listed in Table 3. For the convenience of comparison, we built the SVM models in the original and swap analyses with the expression signatures of 86 probesets proposed by the data contributors [39] and summarized the prediction results (Table 3). In general, our model performed similarly to the 86-probeset model in the original analysis, while the MCC achieved by our model with the validation set in the swap 
TABLE 3: The results of predicting the overall survival milestone outcome of acute myeloma leukemia in original and swap analyses.

\begin{tabular}{lccccccccccc}
\hline & & \multicolumn{3}{c}{ Our model } & \multicolumn{4}{c}{ 86-probe-set model } \\
& & SP & SE & ACC & MCC & AUC & SP & SE & ACC & MCC & AUC \\
\hline \multirow{2}{*}{ Original analysis } & Training & 0.697 & 0.837 & 0.776 & 0.542 & 0.776 & 0.758 & 0.733 & 0.743 & 0.486 & 0.746 \\
& Validation & 0.574 & 0.600 & 0.584 & 0.170 & 0.587 & 0.362 & 0.800 & 0.532 & 0.172 & 0.581 \\
\hline \multirow{2}{*}{ Swap analysis } & Training & 0.830 & 0.700 & 0.779 & 0.533 & 0.765 & 1.000 & 0.433 & 0.779 & 0.564 & 0.717 \\
& Validation & 0.545 & 0.756 & 0.664 & 0.308 & 0.655 & 0.712 & 0.523 & 0.605 & 0.236 & 0.618 \\
\hline
\end{tabular}

In the prediction, high-risk patient was defined as positive sample.

AUC represented the area under the ROC curve.

See notes under Table 2 for more information.

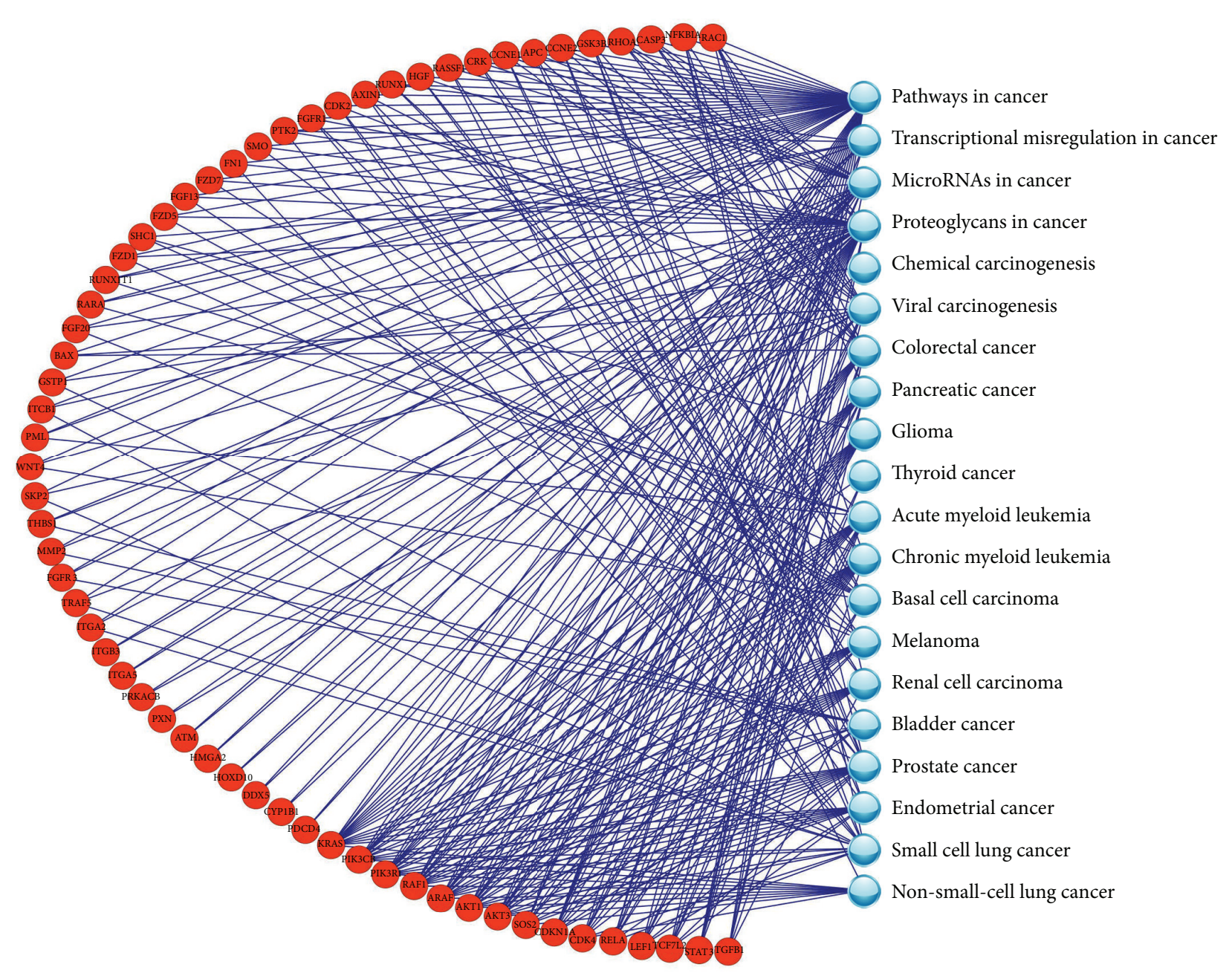

Figure 3: The gene-pathway bipartite network constructed with 62 gene signatures that were used for predicting the molecular subclasses of high-grade glioblastoma.

analysis was 0.308 , which was higher than that achieved by the 86-probeset model.

3.3. Model Performance on Predicting the Molecular Subclasses of High-Grade Glioblastoma. For the high-grade glioblastoma data set, 2712 genes with $P$ value $<0.05$ were selected as DEGs and 62 of them with scores $\geq 1$ were used to construct the SVM models. The gene-pathway bipartite network of 62
DEGs connected to the 20 cancer-related KEGG pathways was shown in Figure 3. The gene KRAS was ranked 1st in the DEG list and had the most connections to the cancer-related pathways. The best parameters of $c$ and $\sigma$ optimized for SVM models in original analysis were 8 and 0.00781 , respectively, and were 0.5 and 0.125 in swap analysis, respectively. The prediction results in original and swap analyses were listed in Table 4. In the original analysis, the prediction accuracy 
TABLE 4: The results of predicting the molecular subclasses of high-grade glioblastoma in original and swap analyses.

\begin{tabular}{lcccccc}
\hline & & \multicolumn{2}{c}{ Our model } & ACC & AUC \\
\hline \multirow{2}{*}{ Original analysis } & Training & 0.900 & 0.900 & 0.900 & 0.800 & 0.900 \\
& Validation & 0.750 & 1.000 & 0.875 & 0.775 & 0.875 \\
\hline \multirow{2}{*}{ Swap analysis } & Training & 0.950 & 0.900 & 0.925 & 0.851 & 0.925 \\
& Validation & 0.800 & 0.567 & 0.683 & 0.377 \\
\hline
\end{tabular}

In the prediction, the gene expression profile termed ProNeural (PN) was defined as positive sample.

AUC represented the area under the ROC curve.

See notes under Table 2 for more information.

for the validation set was as high as $87.5 \%$ and similar to that achieved in the training procedure, indicating the superior performance of the SVM model in predicting the molecular subclasses of high-grade glioblastoma. In the swap analysis, the MCC for validation set was dropped to 0.377 . This was mainly because the number of samples for the model construction was limited.

\section{Discussion}

In clinical researches, the microarray-based gene expression profiling is often used to construct the models for predicting cancer prognosis. Identifying the DEGs accurately plays a key role in the procedure of clinical model construction. Current statistical methods and machine learning algorithms used for DEG selection only focus on the changes of the gene expression levels between the two groups of clinical samples instead of the causes behind these changes and subsequently result in a number of false positive genes unrelated to the phenotypic differences involved in the DEG list and the predictive models becoming unreliable. In our current study, we described a weighted method, which scored each of the genes according to their connections to the cancer-related pathways in the gene-pathway bipartite network, for the purpose of refining the DEG list generated by the statistical methods. By considering the two facts of (1) how many genes connected to a specific pathway and (2) how many pathways involved a specific gene, all the DEGs in the bipartite network were scored by the weighted method. The DEGs with scores $\geq 1$ were considered as the specific cancer-related genes and used to construct the predictive models.

In order to validate the performance of the predictive models, the gene expression data of the clinical samples were collected in our study to predict three clinical endpoints, namely, the reoperative treatment response of breast cancer, the overall survival milestone outcome of acute myeloma leukemia, and the molecular subclasses of highgrade glioblastoma. For the prediction of reoperative treatment response of breast cancer, 29 DEGs were selected from the bipartite network as features to construct SVM models. In both original and swap analyses, our model performed ( $\mathrm{MCC}=0.332$ and 0.343 , resp.) better than the MAQC-II candidate model (MCC $=0.301$ and 0.267, resp.). Moreover, in the swap analysis, the MCC achieved by our model in training procedure $(\mathrm{MCC}=0.343$ ) was equal to that achieved in validation procedure, indicating the robust model performance. When predicting the overall survival milestone outcome of acute myeloma leukemia, the performance of our model with 50 DEGs was similar to that of the 86-probeset model in original analysis. In the swap analysis, the MCC achieved by our model ( $\mathrm{MCC}=0.308$ ) was higher than that $(\mathrm{MCC}=0.236$ ) achieved by the 86 -probeset model. As to the prediction of the molecular subclasses of highgrade glioblastoma, 62 DEGs were used for SVM model construction. The accuracy achieved in the original analysis was as high as $87.5 \%$. Meanwhile, the model performance was robust in the original analysis $(\mathrm{MCC}=0.800$ and 0.775 in training and validation procedures, resp.). Note that, in the swap analysis, the MCC for validation set was only 0.37 . This was mainly because the number of samples used for model construction was limited. In the swap analysis, only 40 samples were used to construct the predictive model, which was insufficient to ensure the reliability of the predictive model.

\section{Conclusions}

In this study, we suggested a strategy to identify the gene signatures, which not only were differentially expressed between two groups of clinical samples but also highly correlated with a specific cancer, from a gene-pathway bipartite network. The predictive models constructed with these gene signatures performed better than those models reported in previous studies. Moreover, in both original and swap analyses, our models achieved similar prediction results, indicating the robust model performance on predicting the cancer prognosis.

\section{Conflict of Interests}

The authors declare that there is no conflict of interests regarding the publication of this paper.

\section{Acknowledgment}

This work was supported by the National Nature Science Foundation of China (nos. 21205085 and 31370060).

\section{References}

[1] L. J. van’t Veer, H. Dai, M. J.van de Vijver et al., “Gene expression profiling predicts clinical outcome of breast cancer," Nature, vol. 415 , no. 6871, pp. 530-536, 2002. 
[2] E. L. Leung, Z.-W. Cao, Z.-H. Jiang, H. Zhou, and L. Liu, "Network-based drug discovery by integrating systems biology and computational technologies," Briefings in Bioinformatics, vol. 14, no. 4, pp. 491-505, 2013.

[3] F. Zhan, J. Hardin, B. Kordsmeier et al., "Global gene expression profiling of multiple myeloma, monoclonal gammopathy of undetermined significance, and normal bone marrow plasma cells," Blood, vol. 99, no. 5, pp. 1745-1757, 2002.

[4] K. R. Hess, K. Anderson, W. F. Symmans et al., "Pharmacogenomic predictor of sensitivity to preoperative chemotherapy with paclitaxel and fluorouracil, doxorubicin, and cyclophosphamide in breast cancer," Journal of Clinical Oncology, vol. 24, no. 26, pp. 4236-4244, 2006.

[5] J. D. Shaughnessy Jr., F. Zhan, B. E. Burington et al., "Avalidated gene expression model of high-risk multiple myeloma is defined by deregulated expression of genes mapping to chromosome 1," Blood, vol. 109, no. 6, pp. 2276-2284, 2007.

[6] H. S. Phillips, S. Kharbanda, R. Chen et al., "Molecular subclasses of high-grade glioma predict prognosis, delineate a pattern of disease progression, and resemble stages in neurogenesis," Cancer Cell, vol. 9, no. 3, pp. 157-173, 2006.

[7] Y. Lee, A. Scheck, T. Cloughesy et al., "Gene expression analysis of glioblastomas identifies the major molecular basis for the prognostic benefit of younger age," BMC Medical Genomics, vol. 1 , no. 1 , article 52, 2008.

[8] A. Oberthuer, F. Berthold, P. Warnat et al., "Customized oligonucleotide microarray gene expression-based classification of neuroblastoma patients outperforms current clinical risk stratification," Journal of Clinical Oncology, vol. 24, no. 31, pp. 5070-5078, 2006.

[9] L. Shi, G. Campbell, W. D. Jones et al., "The MicroArray Quality Control (MAQC)-II study of common practices for the development and validation of microarray-based predictive models," Nature Biotechnology, vol. 28, no. 8, pp. 827-838, 2010.

[10] M. A. van Driel, K. Cuelenaere, P. P. C. W. Kemmeren, J. A. M. Leunissen, and H. G. Brunner, "A new web-based data mining tool for the identification of candidate genes for human genetic disorders," European Journal of Human Genetics, vol. 11, no. 1, pp. 57-63, 2003.

[11] S. Aerts, D. Lambrechts, S. Maity et al., "Gene prioritization through genomic data fusion," Nature Biotechnology, vol. 24, no. 5, pp. 537-544, 2006.

[12] K. Goh, M. E. Cusick, D. Valle, B. Childs, M. Vidal, and A. Barabási, "The human disease network," Proceedings of the National Academy of Sciences of the United States of America, vol. 104, no. 21, pp. 8685-8690, 2007.

[13] E. Wang, A. Lenferink, and M. O'Connor-McCourt, "Genetic studies of diseases - Cancer systems biology: Exploring cancerassociated genes on cellular networks," Cellular and Molecular Life Sciences, vol. 64, no. 14, pp. 1752-1762, 2007.

[14] Y. Moreau and L. C. Tranchevent, "Computational tools for prioritizing candidate genes: boosting disease gene discovery," Nature Reviews Genetics, vol. 13, no. 8, pp. 523-536, 2012.

[15] J. I. F. Bass, A. Diallo, and J. Nelson, "Corrigendum: using networks to measure similarity between genes: association index selection," Nature Methods, vol. 11, no. 3, pp. 349-349, 2014.

[16] P. Hernández, J. Huerta-Cepas, D. Montaner et al., "Evidence for systems-level molecular mechanisms of tumorigenesis," BMC Genomics, vol. 8, article 185, 2007.
[17] F. M. Lopes and J. Cesar, "Gene expression complex networks: synthesis, identification, and analysis," Journal of Computational Biology, vol. 18, no. 10, pp. 1353-1367, 2011.

[18] O. Rozenblatt-Rosen, R. C. Deo, M. Padi et al., "Interpreting cancer genomes using systematic host network perturbations by tumour virus proteins," Nature, vol. 487, no. 7408, pp. 491-495, 2012.

[19] T. Wang, J. Gu, J. Yuan, R. Tao, Y. Li, and S. Li, "Inferring pathway crosstalk networks using gene set co-expression signatures," Molecular BioSystems, vol. 9, no. 7, pp. 1822-1828, 2013.

[20] W. Jiang, X. Li, S. Q. Rao et al., "Constructing disease-specific gene networks using pair-wise relevance metric: application to colon cancer identifies interleukin 8 , desmin and enolase 1 as the central elements," BMC Systems Biology, vol. 2, article 72, 2008.

[21] L. Li, K. Zhang, J. Lee, S. Cordes, D. P. Davis, and Z. Tang, "Discovering cancer genes by integrating network and functional properties," BMC Medical Genomics, vol. 2, no. 1, article 61, 2009.

[22] T. Milenković, V. Memišević, A. K. Ganesan, and N. Pršulj, "Systems-level cancer gene identification from protein interaction network topology applied to melanogenesis-related functional genomics data," Journal of the Royal Society Interface, vol. 7, no. 44, pp. 423-437, 2010.

[23] G. Östlund, M. Lindskog, and E. L. L. Sonnhammer, "Networkbased identification of novel cancer genes," Molecular and Cellular Proteomics, vol. 9, no. 4, pp. 648-655, 2010.

[24] L. Agnelli, M. Forcato, F. Ferrari et al., "The reconstruction of transcriptional networks reveals critical genes with implications for clinical outcome of multiple myeloma," Clinical Cancer Research, vol. 17, no. 23, pp. 7402-7412, 2011.

[25] J. Ahn, Y. Yoon, C. Park, E. Shin, and S. Park, "Integrative gene network construction for predicting a set of complementary prostate cancer genes," Bioinformatics, vol. 27, no. 13, pp. 18461853, 2011.

[26] L. Chen, J. Xuan, R. B. Riggins, R. Clarke, and Y. Wang, "Identifying cancer biomarkers by network-constrained support vector machines," BMC Systems Biology, vol. 5, article 161, 2011.

[27] J. X. Wang, G. Chen, M. Li, and Y. Pan, "Integration of breast cancer gene signatures based on graph centrality," BMC Systems Biology, vol. 5, no. 3, article S10, 2011.

[28] M. D'Antonio, V. Pendino, S. Shruti, and F. D. Ciccarelli, "Network of Cancer Genes (NCG 3.0): integration and analysis of genetic and network properties of cancer genes," Nucleic Acids Research, vol. 40, no. 1, pp. D978-D983, 2012.

[29] J. Roy, C. Winter, Z. Isik, and M. Schroeder, "Network information improves cancer outcome prediction," Briefings in Bioinformatics, 2012.

[30] C. Staiger, S. Cadot, R. Kooter et al., "A critical evaluation of network and pathway-based classifiers for outcome prediction in breast cancer," PLoS ONE, vol. 7, no. 4, Article ID e34796, 2012.

[31] C. Winter, G. Kristiansen, S. Kersting et al., "Google goes cancer: improving outcome prediction for cancer patients by network-based ranking of marker genes," PLoS Computational Biology, vol. 8, no. 5, Article ID e1002511, 2012.

[32] C. Wu, D. D’Argenio, S. Asgharzadeh, and T. Triche, "TARGETgene: a tool for identification of potential therapeutic targets in cancer," PLoS ONE, vol. 7, no. 8, Article ID e43305, 2012.

[33] G. Wu and L. Stein, "A network module-based method for identifying cancer prognostic signatures," Genome Biology, vol. 13, no. 12, article R112, 2012. 
[34] Y. Chen, J. Hao, W. Jiang et al., "Identifying potential cancer driver genes by genomic data integration," Scientific Reports, vol. 3, p. 3538, 2013.

[35] Y. Nie and J. Yu, "Mining breast cancer genes with a network based noise-tolerant approach," BMC Systems Biology, vol. 7, article 49, 2013.

[36] H. Fröhlich, "Including network knowledge into Cox regression models for biomarker signature discovery," Biometrical Journal, vol. 56, no. 2, pp. 287-306, 2014.

[37] L. Jiang, L. Huang, Q. Kuang et al., "Improving the prediction of chemotherapeutic sensitivity of tumors in breast cancer via optimizing the selection of candidate genes," Computational Biology and Chemistry, vol. 49, pp. 71-78, 2014.

[38] A. Chaiboonchoe, S. Samarasinghe, D. Kulasiri, and K. SalehiAshtiani, "Integrated analysis of gene network in childhood leukemia from microarray and pathway databases," BioMed Research International, vol. 2014, Article ID 278748, 7 pages, 2014.

[39] K. H. Metzeler, M. Hummel, C. D. Bloomfield et al., "An 86-probe-set gene-expression signature predicts survival in cytogenetically normal acute myeloid leukemia," Blood, vol. 112, no. 10, pp. 4193-4201, 2008.

[40] J. Li, N. Zhang, Z. Liu, and G. Zhao, "Based on bipartite graph label gene extraction algorithm of network structure," International Journal of Biology, vol. 3, no. 4, p. 64, 2011.

[41] P. Holme, F. Liljeros, C. R. Edling, and B. J. Kim, "Network bipartivity," Physical Review E: Statistical, Nonlinear, and Soft Matter Physics, vol. 68, no. 5, part 2, Article ID 056107, 2003.

[42] T. Zhou, J. Ren, M. Medo, and Y. Zhang, "Bipartite network projection and personal recommendation," Physical Review E, vol. 76, no. 4, Article ID 046115, 2007.

[43] V. N. Vapnik, The Nature of Statistical Learning Theory, Springer, New York, NY, USA, 1995.

[44] V. N. Vapnik, Statistical Learning Theory, Wiley, New York, NY, USA, 1998.

[45] C. Chang and C. Lin, "LIBSVM: a Library for support vector machines," ACM Transactions on Intelligent Systems and Technology, vol. 2, no. 3, article 27, 2011. 

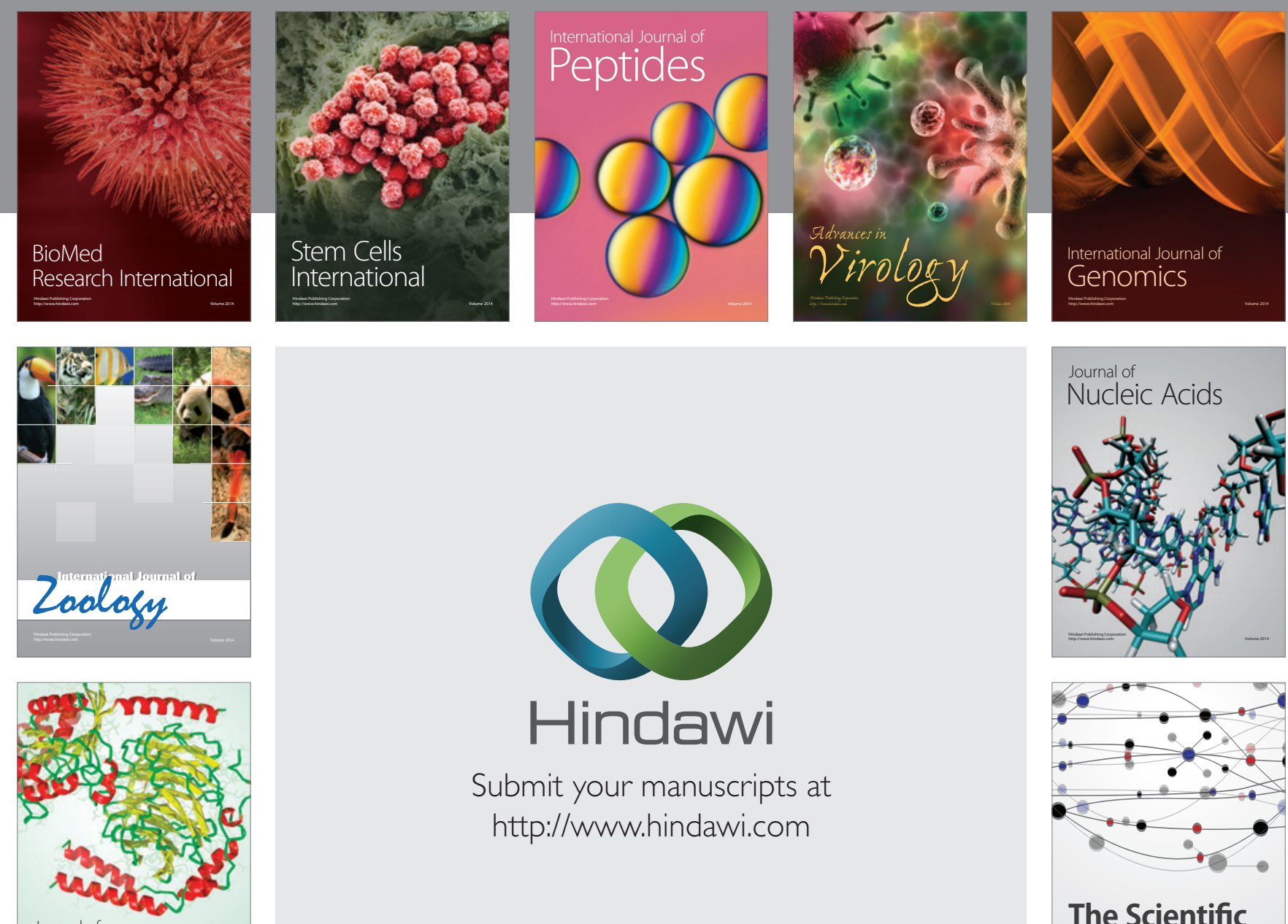

Submit your manuscripts at

http://www.hindawi.com

Journal of
Signal Transduction
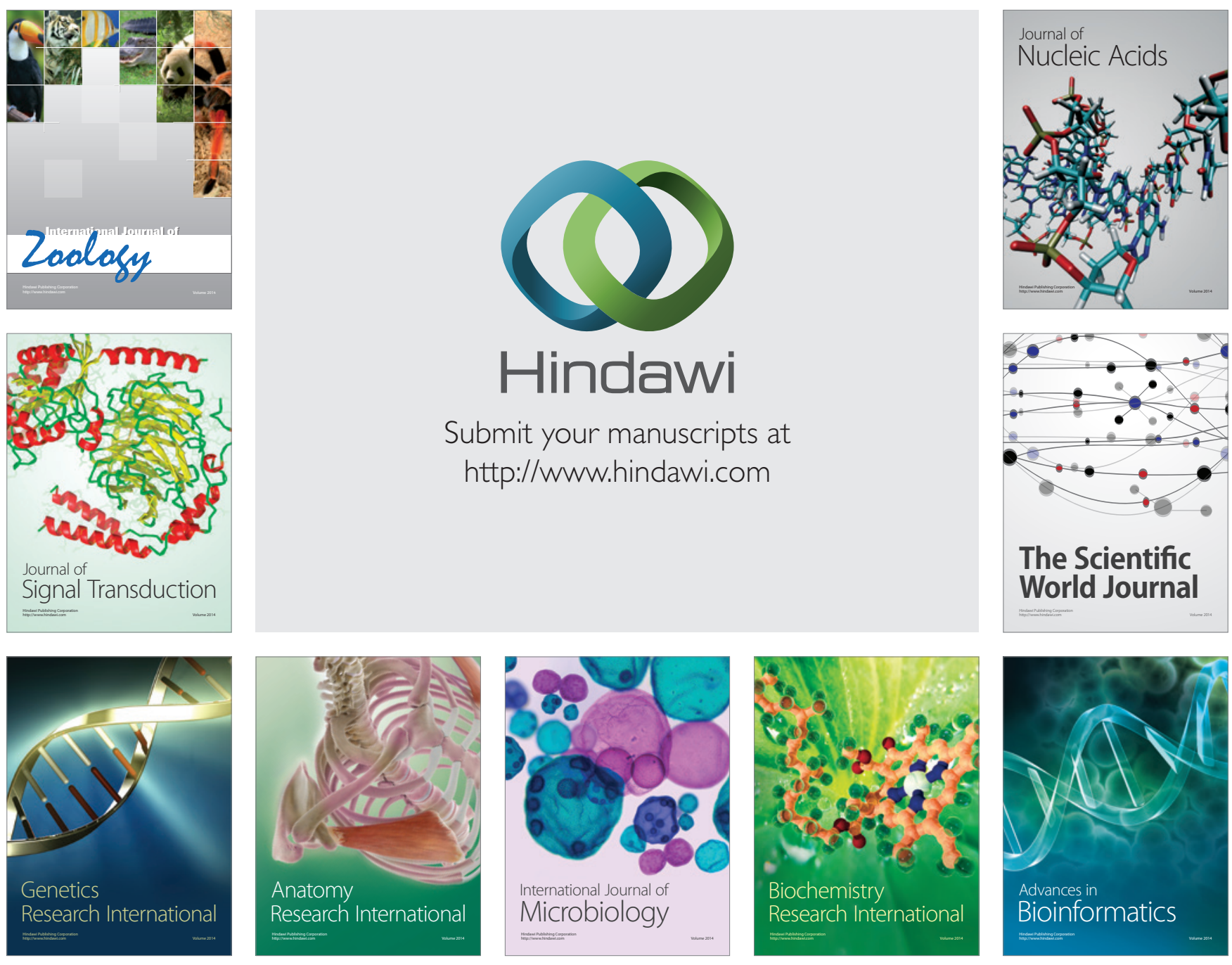

The Scientific World Journal
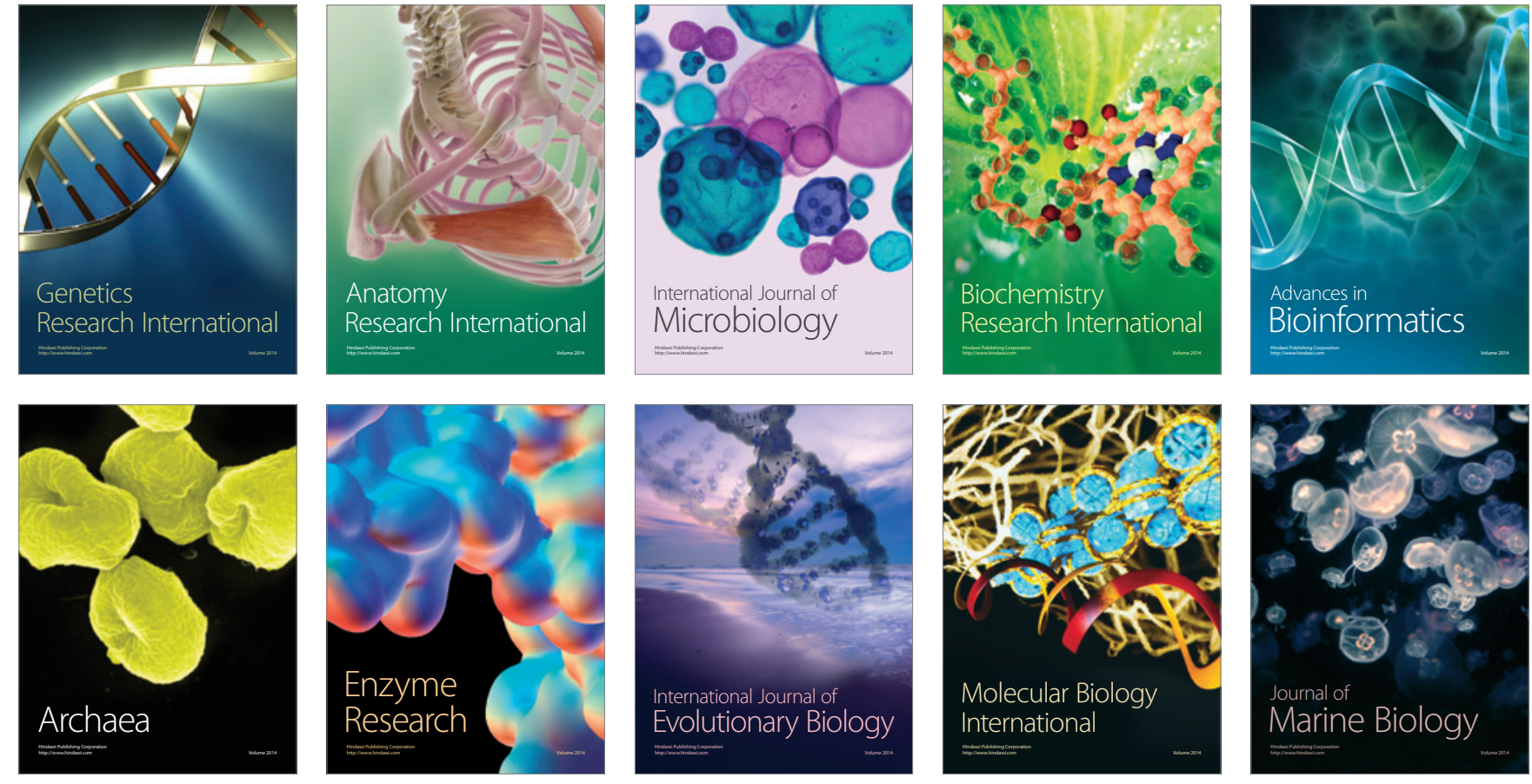